Canad. Math. Bull. Vol. 20 (4), 1977

\title{
SOME GENERALIZATIONS OF AN IDENTITY OF SUBHANKULOV
}

\author{
BY \\ D. SURYANARAYANA* AND DAVID T. WALKER
}

Abstract. In 1957, M. A. Subhankulov established the following identity

$$
\sum_{r=1}^{\infty} \frac{\mu\left(r_{1} r_{2}\right) r_{2}^{2}}{J_{2}(r)} \sum_{d \mid(n, r)} d \mu\left(\frac{r}{d}\right)=\mu^{2}(n) \frac{\pi^{2}}{6}
$$

where $r=r_{1} r_{2}^{2},\left(r_{1}, r_{2}\right)=1 ; \mu$ is the Möbius function and $J_{2}$ is the Jordan totient function of order 2. Since the Ramanujan trigonometrical sum $C(n, r)=\sum_{d \mid(n, r)} d \mu(r / d)$, we rewrite the above identity using $C(n, r)$.

In this paper, we give a generalization of Ramanujan's sum, which generalizes some of the earlier generalizations mainly due to $\mathrm{E}$. Cohen, and prove a theorem from which we deduce some generalizations of the above identity.

§1. Introduction. In 1957, M. A. Subhankulov [10] established the following curious identity (in a slightly different form and notation):

$$
\sum_{r=1}^{\infty} \frac{\mu\left(r_{1} r_{2}\right) r_{2}^{2}}{J_{2}(r)} \sum_{d \mid(n, r)} d \mu\left(\frac{r}{d}\right)=\mu^{2}(n) \frac{\pi^{2}}{6},
$$

where $r=r_{1} r_{2}^{2},\left(r_{1}, r_{2}\right)=1 ; \mu$ is the well-known Möbius function and $J_{2}$ is the Jordan totient function of order 2. The identity (1) may also be found in a subsequent paper of Subankulov written jointly with S. N. Muhatarov (cf. [11], eq. (3)).

Since it is known (cf. [8], Theorem 271) that

$$
C(n, \tilde{i})=\sum_{d \mid(n, r)} d \mu\left(\frac{r}{d}\right)
$$

Received by the editors Jan. 4, 1977

AMS (MOS) Subject classifications. Primary 10A20, 10A99.

Key words and phrases: Möbius function, Jordan totient function, generalized Ramanujan's sum, $k$-free integers, Riemann zeta function.

* On leave from Andhra University, Waltair, India. 
and $\zeta(2)=\pi^{2} / 6$, where $\zeta(s)$ is the Riemann Zeta function defined for $s>1$ by $\zeta(s)=\sum_{n=1}^{\infty} n^{-s}$ and $C(n, r)$ is the Ramanujan trigonometrical sum (cf. [8], $\S 5.6)$ defined by

$$
C(n, r)=\sum_{\substack{x(\bmod r) \\(x, r)=1}} \exp (2 \pi i x n / r),
$$

we can rewrite the identity (1) as follows:

$$
\sum_{r=1}^{\infty} \frac{\mu\left(r_{1} r_{2}\right) r_{2}^{2}}{J_{2}(r)} C(n, r)=\mu^{2}(n) \zeta(2) .
$$

In this paper, we establish some identities as generalizations of the identity (4). For example, we prove that for $k \geq 1$,

$$
\sum_{r=1}^{\infty} \frac{\mu\left(r_{1} r_{2}\right) r_{2}^{2 k}}{J_{2 k}(r)} C_{k}(n, r)=q_{2 k}(n) \zeta(2 k),
$$

where $r=r_{1} r_{2}^{2},\left(r_{1}, r_{2}\right)=1 ; J_{k}(r)$ is the Jordan totient function of order $k$ (cf. [7], p. 147; also cf. [2] and [3]) which has the arithmetic evaluation

$$
J_{k}(r)=\sum_{\left.d\right|_{r}} d^{k} \mu\left(\frac{r}{d}\right)=r^{k} \prod_{\left.p\right|_{r}}\left(1-p^{-k}\right),
$$

$p$ a prime, $q_{k}(r)=1$ or 0 according as $r \in Q_{k}$ or $r \notin Q_{k}, Q_{k}$ being the set of all $k$-free integers (a positive integer $r$ is called $k$-free, if $r$ is not divisible by $p^{k}$ for any prime $p)$ and $C_{k}(n, r)$ is E. Cohen's [1] generalized Ramanujan sum defined by

$$
C_{k}(n, r)=\sum_{\substack{x\left(\bmod r^{k}\right) \\\left(x, r^{k}\right)_{k}=1}} \exp \left(2 \pi i x n / r^{k}\right)
$$

the summation being extended over all $x$ modulo $r^{k}$, whose greatest common $k$ th power divisor with $r^{k}$ is 1. E. Cohen (cf. [1], eq. (2.5)) also established the following arithmetic evaluation of $C_{k}(n, r)$ :

$$
C_{k}(n, r)=\sum_{\substack{d^{k}|n \\ d| r}} d^{k} \mu\left(\frac{r}{d}\right)
$$

In fact, we first prove a general result, from which we deduce some generalizations of the identity (4) (for example, see Remark 2 and (16) of §3), in the following: 
THEOREM. If $\alpha$ and $\beta$ are integers each $\geq 2$; if $k$, $u$, and $n_{1}, \ldots, n_{u}$ are integers each $\geq 1$; and if $v_{p}(n)$ is the non-negative integer such that $p^{v_{p}(n)}$ is the highest power of the prime $p$ that divides $n$, where $n=\left(n_{1}, \ldots, n_{u}\right)$, then

(9) $\sum_{r=1}^{\infty} \frac{\mu\left(r_{1} r_{2}\right) r_{2}^{\alpha(\beta-1)}}{J_{\alpha}(r)} C_{k}^{(u)}\left(n_{1}, \ldots, n_{u}, r\right)$

$$
\begin{aligned}
= & \zeta(\alpha) \prod_{\substack{p \\
k \leq v_{p}(n)<(\beta-1) k}}\left(1-\frac{1}{p^{\alpha-k u}}\right) \prod_{\substack{p \\
(\beta-1) k \leq v_{p}(n)<\beta k}}\left(1-\frac{1}{p^{\alpha-k u}}+\frac{1}{p^{\alpha-(\beta-1) k u}}\right) \\
& \times \prod_{\substack{p \\
v_{p}(n) \geq \beta k}}\left(1-\frac{1}{p^{\alpha-k u}}+\frac{1}{p^{\alpha-(\beta-1) k u}}-\frac{1}{p^{\alpha-\beta k u}}\right),
\end{aligned}
$$

where $r=r_{1} r_{2}^{\beta},\left(r_{1}, r_{2}\right)=1$, and each product is extended over all primes $p$ subject to the restrictions on $v_{p}(n)$ mentioned under each product.

In the above Theorem $C_{k}^{(u)}\left(n_{1}, \ldots, n_{u}, r\right)$ is a generalization of Ramanujan's sum defined as follows:

$$
C_{k}^{(u)}\left(n_{1}, \ldots, n_{u}, r\right)=\sum_{\substack{\left(x_{i}\right)\left(\bmod r^{k}\right) \\\left(\left(x_{i}\right), r^{k}\right)_{k}=1}} \exp \left(2 \pi i\left(n_{1} x_{1}+\cdots+n_{u} x_{u}\right) / r^{k}\right)
$$

where the summation is extended over all $x_{i}$ modulo $r^{k}$, for $i=1, \ldots, k$, such that the greatest common $k$ th power divisor of $\left(x_{1}, \ldots, x_{u}\right)$ and $r^{k}$ is 1 . Following the method adopted by M. Sugunamma (cf. [12]), Theorem 1) and E. Cohen (cf. [5], Lemma 2 and cf. [6], p. 30), we get the following arithmetic evaluation:

$$
C_{k}^{(u)}\left(n_{1}, \ldots, n_{u}, r\right)=\sum_{\substack{d^{k}|n \\ d| r}} d^{k u} \mu\left(\frac{r}{d}\right)
$$

where $n=\left(n_{1}, \ldots, n_{u}\right)$.

REMARK 1. We note here that (10) gives a generalization of some of the known generalizations of Ramanujan's sum. For example, when $n_{1}=\cdots=$ $n_{u}=n,(10)$ reduces to $C_{k}^{(u)}(n, r)$, which is due to M. Sugunamma [12]; when $u=1, n_{1}=n,(10)$ reduces to $C_{k}(n, r)$ which is due to E. Cohen [1]; when $k=1$, $n_{1}=\cdots=n_{u}=n,(10)$ reduces to $C^{(u)}(n, r)$ which is again due to E. Cohen [4]; and finally when $k=1,(10)$ reduces to $C^{(u)}\left(n_{1}, \ldots, n_{u}, r\right)$ which is once again due to E. Cohen (cf. [5] and [6]).

§2. Proof of the Theorem. Since the general term in the series (9) is a multiplicative function of $r$ and the series is absolutely convergent, it can be expanded into an infinite product of Euler type (cf. [8],Theorem 286). Hence 
by (6) and (11), we have

$$
\begin{aligned}
& \sum_{r=1}^{\infty} \frac{\mu\left(r_{1} r_{2}\right) r_{2}^{\alpha(\beta-1)}}{J_{\alpha}(r)} C_{k}^{(u)}\left(u_{1}, \ldots, n_{u}, r\right) \\
& =\prod_{p}\left\{1-\frac{C_{k}^{(u)}\left(n_{1}, \ldots, n_{u}, p\right)}{J_{\alpha}(p)}-\frac{C_{k}^{(u)}\left(n_{1}, \ldots, n_{u}, p^{\beta}\right) p^{\alpha(\beta-1)}}{J_{\alpha}\left(p^{\beta}\right)}\right\} \\
& =\prod_{\substack{p \\
v_{p}(n)<k}}\left(1+\frac{1}{p^{\alpha}-1}\right) \prod_{\substack{p \\
k \leq v_{p}(n)<(\beta-1) k}}\left(1-\frac{p^{k u}-1}{p^{\alpha}-1}\right) \\
& \times \prod_{\substack{p \\
(\beta-1) k \leq v_{p}(n)<\beta k}}\left(1-\frac{p^{k u}-1}{p^{\alpha}-1}+\frac{p^{(\beta-1) k u}}{p^{\alpha}-1}\right) \prod_{\substack{p \\
v_{p}(n) \geq \beta k}}\left(1-\frac{p^{k u}-1}{p^{\alpha}-1}-\frac{p^{\beta k u}-p^{(\beta-1) k u}}{p^{\alpha}-1}\right) \\
& =\prod_{\substack{p \\
v_{p}(n)<k}}\left(\frac{1}{1-p^{-\alpha}}\right) \prod_{\substack{p \\
k \leq v_{p}(n)<(\beta-1) k}}\left(\frac{1-p^{-\alpha+k u}}{1-p^{-\alpha}}\right) \prod_{\substack{p \\
(\beta-1) k \leq v_{p}(n)<\beta k}}\left(\frac{1-p^{-\alpha+k u}+p^{-\alpha+(\beta-1) k u}}{1-p^{-\alpha}}\right) \\
& \times \prod_{\substack{p \\
v_{p}(n) \geq \beta k}}\left(\frac{1-p^{-\alpha+k u}+p^{-\alpha+(\beta-1) k u}-p^{-\alpha+\beta k u}}{1-p^{-\alpha}}\right) \\
& =\prod_{p}\left(\frac{1}{1-p^{-\alpha}}\right) \prod_{\substack{p \\
k \leq v_{\mathrm{p}}(n)<(\beta-1) k}}\left(1-\frac{1}{p^{\alpha-k u}}\right) \prod_{\substack{p \\
(\beta-1) k \leq v_{p}(n)<\beta k}}\left(1-\frac{1}{p^{\alpha-k u}}+\frac{1}{p^{\alpha-(\beta-1) k u}}\right) \\
& \times \prod_{\substack{p \\
v_{p}(n) \geq \beta k}}\left(1-\frac{1}{p^{\alpha-k u}}+\frac{1}{p^{\alpha-(\beta-1) k u}}-\frac{1}{p^{\alpha-\beta k u}}\right) .
\end{aligned}
$$

Now, applying Euler's result that $\zeta(\alpha)=\Pi_{p}\left(1-p^{-\alpha}\right)$, (cf. [8], Theorem 280), the Theorem follows.

§3. Some special cases. Taking $\beta=2$ in (9), we have the following result: For $\alpha \geq 2, k \geq 1$ and $u \geq 1$,

$$
\sum_{r=1}^{\infty} \frac{\mu\left(r_{1} r_{2}\right) r_{2}^{\alpha}}{J_{\alpha}(r)} C_{k}^{(u)}\left(n_{1}, \ldots, n_{u}, r\right)=\zeta(\alpha) \prod_{p^{2 k} / n}\left(1-\frac{1}{p^{\alpha-2 k u}}\right)
$$

where $r=r_{1} r_{2}^{2},\left(r_{1}, r_{2}\right)=1$ and $n=\left(n_{1}, \ldots, n_{u}\right)$.

If $n \in Q_{2 k}$, then the right side of (12) becomes $\zeta(\alpha)$. On the other hand, if $n \notin Q_{2 k}$ and $\alpha=2 k u$, then the right side of (12) becomes zero. Hence from (12), we obtain the following:

$$
\sum_{r=1}^{\infty} \frac{\mu\left(r_{1} r_{2}\right) r_{2}^{\alpha}}{J_{\alpha}(r)} C_{k}^{(u)}\left(n_{1}, \ldots, n_{u}, r\right)=\left\{\begin{array}{rll}
\zeta(\alpha), & \text { if } & n \in Q_{2 k} \\
0, & \text { if } & n \notin Q_{2 k}
\end{array} \text { and } \alpha=2 k u\right.
$$

Taking $\alpha=2 k u$ in (13), we have

$$
\sum_{r=1}^{\infty} \frac{\mu\left(r_{1} r_{2}\right) r_{2}^{2 k u}}{J_{2 k u}(r)} C_{k}^{(u)}\left(n_{1}, \ldots, n_{u}, r\right)=q_{2 k}(n) \zeta(2 k u)
$$


As a particular case of (14), taking $n_{1}=\cdots=n_{u}=n$, we have the identity

$$
\sum_{r=1}^{\infty} \frac{\mu\left(r_{1} r_{2}\right) r_{2}^{2 k u}}{J_{2 k u}(r)} C_{k}^{(u)}(n, r)=q_{2 k}(n) \zeta(2 k u)
$$

Remark 2. Now, taking $u=1$ in (15), we have the identity (5). Also, taking $k=1$ in (15), we have the following identity, which is a generalization of (4), different from (5):

$$
\sum_{r=1}^{\infty} \frac{\mu\left(r_{1} r_{2}\right) r_{2}^{2 u}}{J_{2 u}(r)} C^{(u)}(n, r)=\mu^{2}(n) \zeta(2 u)
$$

Taking $\alpha=2 k(u+1)$ in (12), we get the following identity:

$$
\sum_{r=1}^{\infty} \frac{\mu\left(r_{1} r_{2}\right) r_{2}^{2 k(u+1)}}{J_{2 k(u+1)}(r)} C_{k}^{(u)}\left(n_{1}, \ldots, n_{u}, r\right)=\zeta(2 k(u+1)) \frac{\Phi_{2 k}(n)}{n},
$$

where $\Phi_{k}(n)$ is Klee's [9] generalized Euler totient function $\varphi(n)$, which has the arithmetic evaluation

$$
\Phi_{k}(n)=\sum_{d^{k} \mid n} \mu(d)\left(n / d^{k}\right)=n \prod_{p^{k} / n}\left(1-p^{-k}\right) .
$$

As it is clear from (6) and (18) that $\Phi_{k}\left(n^{k}\right)=J_{k}(n)$, we obtain, from (17), the following identity:

$$
\sum_{r=1}^{\infty} \frac{\mu\left(r_{1} r_{2}\right) r_{2}^{2 k(u+1)}}{J_{2 k(u+1)}(r)} C_{k}^{(u)}\left(n_{1}^{2 k}, \ldots, n_{u}^{2 k}, r\right)=\zeta(2 k(u+1)) \frac{J_{2 k}(n)}{n^{2 k}}
$$

Taking $n_{1}=\cdots=n_{u}=n$ in (19), we have

$$
\sum_{r=1}^{\infty} \frac{\mu\left(r_{1} r_{2}\right) r_{2}^{2 k(u+1)}}{J_{2 k(u+1)}(r)} C_{k}^{(u)}\left(n^{2 k}, r\right)=\zeta(2 k(u+1)) \frac{J_{2 k}(n)}{n^{2 k}}
$$

As a particular case of (20), taking $k=1$ and $u=1$, we have

$$
\sum_{r=1}^{\infty} \frac{\mu\left(r_{1} r_{2}\right) r_{2}^{4}}{J_{4}(r)} C\left(n^{2}, r\right)=\frac{\pi^{4}}{90}\left(\frac{J_{2}(n)}{n^{2}}\right)
$$

Remark 3. We can deduce some results from the Theorem in case $\beta \geq 3$ also. For example, in this case, if $\alpha=k u$ and $k \leq v_{p}(n)<(\beta-1) k$ for some prime divisor $p$ of $n=\left(n_{1}, \ldots, n_{u}\right)>1$, then the sum of the series in (9) becomes zero.

\section{REFERENCES}

1. E. Cohen, An extension of Ramanujan's sum, Duke Math. J., 16 (1949), 85-90.

2. E. Cohen, Some totient functions, Duke Math. J., 23 (1956), 515-522.

3. E. Cohen, Generalizations of the Euler $\varphi$-function, Scripta Math., 23 (1957), 157-161.

4. E. Cohen, Trigonometric sums in elementary number theory, Amer. Math. Monthly, 66 (1959), 105-117. 
5. E. Cohen, A class of arithmetical functions in several variables with applications to congruences, Trans. Amer. Math. Soc., 96 (1960), 355-381.

6. E. Cohen, A trigonometric sum, Math. Student, 28 (1960), 29-32.

7. L. E. Dickson, History of the theory of numbers, Vol. I, Chelsea Publishing Company, reprinted, 1952.

8. G. H. Hardy and E. M. Wright, An introduction to the theory of numbers, Fourth edition, Oxford University Press, 1960.

9. V. L. Klee, A generalization of Euler's function, Amer. Math. Monthly, 55 (1948), 358-359.

10. M. A. Subhankulov, Some asymptotic formulas in additive theory of numbers (Russian), Scientific Journal of the Tadzhik University, Vol. X, No. 4, (1957), 15-22.

11. M. A. Subhankulov and S. N. Muhatarov, Representations of a number as a sum of two square-free numbers (Russian), Izv. Akad. Nauk UzSSR Ser. Fiz.-Mat. Nauk 1960, No. 4, 3-10.

12. M. Sugunamma, Eckford Cohen's generalizations of Ramanujan's trigonometrical sum C(n, r), Duke Math. J., 27 (1960), 323-330.

Department of Mathematical Sciences

MeMPhis STATE UnIVERSiTY

MeMPHIS, TeNNESSEE 38152 